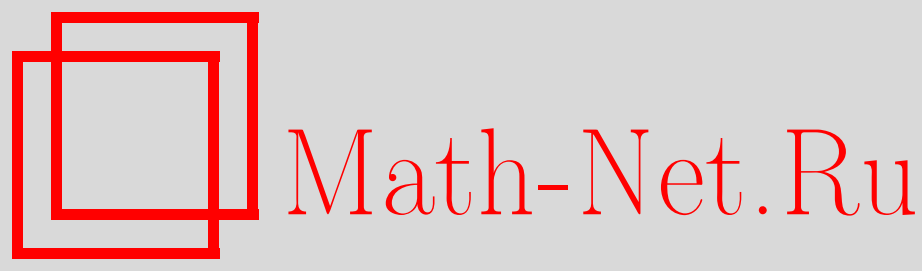

С. П. Суетин, Некоторый аналог вариационных формул Адамара и Шиффера, ТМФ, 2012, том 170, номер 3, 335341

DOI: https://doi.org/10.4213/tmf6770

Использование Общероссийского математического портала Math-Net.Ru подразумевает, что вы прочитали и согласны с пользовательским соглашением http://www.mathnet.ru/rus/agreement

Параметры загрузки:

IP : 54.166 .219 .16

26 апреля 2023 г., $14: 59: 28$

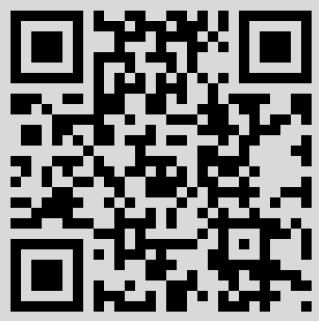




\title{
ФИЗИКА
}

Том 170, № 3

март, 2012

(C) 2012 г.

\section{НЕКОТОРЫЙ АНАЛОГ ВАРИАЦИОННЫХ ФОРМУЛ АДАМАРА И ШИФФЕРА}

\begin{abstract}
Получена достаточно общая вариационная формула для функции Грина, из которой вытекают, в частности, классические вариационные формулы Адамара и Шиффера.
\end{abstract}

Ключевые слова: функция Грина, вариационная формула Адамара, вариационная формула Шиффера.

\section{1. ФОРМУЛА АДАМАРА}

Пусть $G$ - ограниченная конечносвязная область, граница $\gamma$ которой состоит из конечного числа (замкнутых) аналитических кривых, $n_{z}=e^{i \alpha_{z}}-$ единичная внутренняя нормаль к кривой $\gamma$ в точке $z \in \gamma$. Пусть $\psi(s)$ - положительная аналитическая функция натурального параметра на $\gamma$ (при $z \in \gamma$ будем иногда писать $\psi(z))$. Тогда для произвольного достаточно малого $\varepsilon>0$ можно определить положительный сдвиг $\varepsilon \psi(z) n_{z}=\varepsilon \psi(z) e^{i \alpha z}$ внутрь области $G$ по направлению внутренней нормали $n_{z}$ на величину $\delta n_{z}:=\varepsilon \psi(z)>0$. Кривая $\gamma$ переходит в кривую $\gamma^{*}:=\left\{z^{*}=z+\delta n_{z} \cdot n_{z}, z \in \gamma\right\}$, ограничивающую новую область $G^{*}, G^{*} \Subset G$, с границей $\partial G^{*}=\gamma^{*}$.

Обозначим через $g(z, \zeta)$ функцию Грина исходной области $G$ с особенностью в точке $\zeta \in K \Subset G$; пусть $g^{*}(z, \zeta)$ - соответствующая функция Грина для области $G^{*}$. Имеет место следующая вариационная формула Адамара [1]-[4]:

$$
g^{*}(z, \zeta)-g(z, \zeta)=-\frac{1}{2 \pi} \int_{\gamma} \frac{\partial g(w, \zeta)}{\partial n_{w}} \frac{\partial g(w, z)}{\partial n_{w}} \delta n_{w} d s_{w}+O\left(\varepsilon^{2}\right),
$$

где $z \in G^{*}$, причем оценка $O\left(\varepsilon^{2}\right)$ справедлива равномерно по $\zeta \in K \Subset G^{*}$. Поскольку $G^{*} \Subset G$, при $z \in G^{*}$, очевидно, имеем $g^{*}(z, \zeta)<g(z, \zeta)$. Формула (1) дает количественную оценку для последнего неравенства.

Формула Адамара (1) имеет многочисленные приложения, так как через функцию Грина выражаются в конечном итоге все основные функции, связанные с областью $G$ (так называемые "domain-functions"): гармонические меры компонент границы $\gamma$, функция Неймана, я́дра Бергмана и Сегё, соответствующая матрица периодов

* Математический институт им. В. А. Стеклова РАН, Москва, Россия. E-mail: suetin@mi.ras.ru 
(см. прежде всего работу [5], а также [6] и [7]). Тем самым вариации этих функций также могут быть выражены через вариацию функции Грина, а значит, представлены в явном виде с помощью формулы (1) [2], [5], [6], [8]-[13].

Доказательство формулы (1) довольно короткое. Действительно, по формуле Тейлора для $z \in \gamma$ имеем (все дальнейшие соотношения выполняются равномерно по $\zeta \in K \Subset G)$

$$
g\left(z^{*}, \zeta\right)=g(z, \zeta)+\frac{\partial g(z, \zeta)}{\partial n_{z}} \delta n_{z}+O\left(\varepsilon^{2}\right)=\frac{\partial g(z, \zeta)}{\partial n_{z}} \delta n_{z}+O\left(\varepsilon^{2}\right), \quad z \in \gamma .
$$

Следовательно,

$$
\left.g(z, \zeta)\right|_{\gamma^{*}}=g\left(z^{*}, \zeta\right)=\frac{\partial g(z, \zeta)}{\partial n_{z}} \delta n_{z}+O\left(\varepsilon^{2}\right)
$$

и $g(z, \zeta)=-\ln |z-\zeta|+\{$ регулярная часть $\}$ при $z \in G^{*}$. Теперь уже ясно, что́ надо делать: для $w \in \gamma^{*}$ по граничным значениям $u_{0}(w)=\left(\partial g(w, \zeta) / \partial n_{w}\right) \delta n_{w}$ на кривой $\gamma^{*}$ надо с помощью формулы Грина построить гармоническую в области $G^{*}$ функцию $u(z)$ :

$$
u(z)=\frac{1}{2 \pi} \int_{\gamma^{*}} u_{0}(w) \frac{\partial g^{*}(w, z)}{\partial n_{w}} d s_{w}=\frac{1}{2 \pi} \int_{\gamma} \frac{\partial g(w, \zeta)}{\partial n_{w}} \delta n_{w} \frac{\partial g(w, z)}{\partial n_{w}} d s_{w}+O\left(\varepsilon^{2}\right) .
$$

В правой части последнего равенства в (3) мы заменили $\gamma^{*}$ на $\gamma$ и $g^{*}(w, z)$ на $g(w, z)$ с точностью $O\left(\varepsilon^{2}\right)$ на следующем основании. В силу принципа максимума модуля $u(z)=O(\varepsilon)$ равномерно в произвольной области $\Omega \Subset G^{*}$ с гладкой границей. Для $z \in \Omega$ интеграл по кривой $\gamma^{*}$ можно заменить на интеграл по кривой $\partial \Omega$. Теперь уже на $\partial \Omega$ можно заменить ${ }^{1)} g^{*}(w, z)$ на $g(w, z)$ с точностью $O(\varepsilon)$, а затем вернуться к интегрированию по $\gamma^{*}$ (при условии, что $z \in \Omega$ ) и окончательно перейти к интегрированию по $\gamma$ с общей точностью $O\left(\varepsilon^{2}\right)$.

Рассмотрим теперь разность $k(z, \zeta):=g(z, \zeta)-u(z)$. В области $G^{*}$ имеем равенство $k(z, \zeta)=-\ln |z-\zeta|+\{$ регулярная часть $\}$, а на границе

$$
\left.k(z, \zeta)\right|_{\gamma^{*}}=g\left(z^{*}, \zeta\right)-u\left(z^{*}\right)=g\left(z^{*}, \zeta\right)-\frac{\partial g(z, \zeta)}{\partial n_{z}} \delta n_{z}+O\left(\varepsilon^{2}\right)=O\left(\varepsilon^{2}\right) .
$$

Но по определению $g^{*}\left(z^{*}, \zeta\right)=0, z^{*} \in \gamma^{*}$. Таким образом, для гармонической в области $G^{*}$ функции $g^{*}(z, \zeta)-k(z, \zeta)$ имеем $g^{*}(z, \zeta)-k(z, \zeta)=O\left(\varepsilon^{2}\right)$ на $\gamma^{*}$. Следовательно, в силу принципа максимума разность $g^{*}(z, \zeta)-k(z, \zeta)=O\left(\varepsilon^{2}\right)$ всюду в $G^{*}$ (и равномерно по $\zeta \in K \Subset G)$. Отсюда и из соотношения (3) вытекает, что

$$
\begin{aligned}
g^{*}(z, \zeta)-g(z, \zeta) & =-u(z)+O\left(\varepsilon^{2}\right)= \\
& =-\frac{1}{2 \pi} \int_{\gamma} \frac{\partial g(w, \zeta)}{\partial n_{w}} \frac{\partial g(w, z)}{\partial n_{w}} \delta n_{w} d s_{w}+O\left(\varepsilon^{2}\right) .
\end{aligned}
$$

Данная формула и есть вариационная формула Адамара (1). Отметим, что формула Адамара (1) справедлива и при более общих вариациях границы $G$ вдоль нормали к $\gamma$ : границу можно сдвигать вдоль нормали с помощью аналитической функции $\psi(s)$ переменного знака (подробнее см. работы [6], [14]).

1) Из $(2)$ вытекает, что $g^{*}(w, \zeta)-g(w, \zeta)=O(\varepsilon)$ равномерно по $w \in \partial \Omega, \zeta \in K$, и такая же оценка справедлива для нормальных производных. 


\section{2. ФОРМУЛЫ ШИФФЕРА}

Формула Адамара (1) возникает в процессе граничной вариации исходной области $G$. Рассмотрим теперь внутреннюю вариацию Шиффера [2] (см. также [5], [6]):

$$
z^{*}=z+\frac{e^{2 i \phi} \rho^{2}}{z-z_{0}}
$$

где точка $z_{0} \in G, 0 \leqslant \phi<\pi$ и $\rho>0$ - малый параметр. Преобразование (4) конформно и однолистно во внешности круга $\left|z-z_{0}\right|>\rho$ и отображает внешность круга на внешность отрезка $\left[z_{0}-2 \rho e^{i \phi}, z_{0}+2 \rho e^{i \phi}\right]$ длины $4 \rho$ с центром в точке $z_{0}$. Тем самым при достаточно малых $\rho>0$ кривая $\gamma$ конформно и однолистно преобразуется в аналитическую кривую $\gamma^{*}$ того же гомотопического класса, ограничивающую новую область $G^{*}, z_{0} \in G^{*}$. Новую область $G^{*}$ естественно рассматривать как вариацию исходной области $G$, полученную в результате преобразования (4). Хорошо известно, что внутренняя вариация Шиффера (4) приводит к следующей вариации функции Грина [2], [5], [6]:

$$
g^{*}(z, \zeta)-g(z, \zeta)=\operatorname{Re}\left\{e^{2 i \phi} \rho^{2}\left(P^{\prime}\left(z_{0}, \zeta\right) P^{\prime}\left(z_{0}, z\right)-\frac{P^{\prime}(\zeta, z)}{\zeta-z_{0}}-\frac{P^{\prime}(z, \zeta)}{z-z_{0}}\right)\right\}+O\left(\rho^{4}\right),
$$

где $P(w, \zeta)=g(w, \zeta)+i h(w, \zeta)=-\ln (w-\zeta)+U(w, \zeta)-$ комплексная функция Грина, $h(w, \zeta)$ - функция, гармонически сопряженная $g(w, \zeta)$. Функции $h(w, \zeta), P(w, \zeta)-$ многозначные функции в области $D$; производная $P^{\prime}(w, \zeta)$ - однозначная функция (через $P^{\prime}(w, \zeta)$ здесь и далее обозначается производная функции $P(w, \zeta)$ по первому аргументу: $\left.P^{\prime}(w, \zeta)=2 \partial g(w, \zeta) / \partial w\right)$. Формула (5) доказана ниже в разделе 3. Отметим, что эта формула справедлива для произвольной конечносвязной области $G$ с произвольной границей - это доказывается путем предельного перехода (подробнее см. [6], а также раздел 3 настоящей работы). При помощи формулы Тейлора из (5) получаем вторую вариационную формулу Шиффера, в которой учитывается также вариация точек $z, \zeta \in G$ :

$$
g^{*}\left(z^{*}, \zeta^{*}\right)-g(z, \zeta)=\operatorname{Re}\left\{e^{2 i \phi} \rho^{2} P^{\prime}\left(z_{0}, \zeta\right) P^{\prime}\left(z_{0}, z\right)\right\}+O\left(\rho^{4}\right) .
$$

Вариационные формулы Шиффера (5), (6) тоже приводят к явным вариационным формулам для всех основных функций, связанных с областью $G$ [5], [7]. Эти формулы имеют многочисленные применения, прежде всего, в геометрической теории функций комплексного переменного при решении задач, связанных с проблемой модулей, и при описании соответствующей экстремальной метрики (см. работы [15], [16], а также [17]-[19]). В последнее время с помощью вариационных формул удалось доказать существование $S$-кривых для некоторых достаточно общих задач равновесия в теории потенциала, интересных с точки зрения их приложений к различным актуальным задачам математической физики и теории рациональных аппроксимаций аналитических функций [12], [13], [20]-[23].

\section{3. ОБОБЩЕННАЯ ВАРИАЦИЯ}

Пусть теперь $h(z)$ - произвольная функция, голоморфная в некоторой окрестности $V$ кривой $\gamma$. Определим преобразование (вариацию) переменной $z \mapsto z_{t}$ :

$$
z_{t}=z+\operatorname{th}(z)
$$


где $t$ - малый комплексный параметр [12], [20], [22], [24]. Ясно, что при достаточно малом $t,|t| \leqslant \varepsilon_{0}$, преобразование $(7)$ является конформным и однолистным в $V$ и переводит кривую $\gamma$ в новую кривую $\gamma^{*}$, ограничивающую новую область $G^{*}$. Отметим, что формула (7) как частные случаи содержит вариацию Адамара и вариацию Шиффера. Цель настоящей работы - найти формулу достаточно общего характера для вариации функции Грина исходной области $G$ при преобразовании (7) такую, что из нее вытекают вариационные формулы Адамара (1) и Шиффера (5), а также некоторые другие явные формулы, уже нашедшие приложения [12], [13], [20]-[22].

Сдвиг $\Delta z=t h(z)$ от точки $z$ к точке $z^{*}=z_{t}$ происходит теперь не по нормали. Но на $\gamma$ мы имеем $\partial g(z, \zeta) / \partial s_{z}=0$, поэтому вклад в приращение функции Грина в формуле Тейлора все равно дает только сдвиг $\delta n_{z}$ по нормали $n_{z}$. Найдем его выражение через исходный сдвиг $\Delta w=t h(w)$. Пусть $\alpha_{w}$ - направление внутренней по отношению к области $D$ нормали к кривой $\gamma$ в точке $w$ (т. е. угол, который нормаль составляет с осью $x), \beta_{w}$ - направление касательной (т. е. угол, который касательная составляет с осью $x$, отсчитываемый в положительном направлении: область $G$ остается слева при обходе по $\gamma)$. Имеем $\alpha_{w}-\beta_{w}=\pi / 2$ и, следовательно, $\delta n_{w}=\operatorname{Re}\left(e^{-i \alpha_{w}} \Delta w\right)=\operatorname{Re}\left(e^{-i \alpha_{w}} t h(w)\right)$. Поступим теперь так же, как в разделе 1. Тогда по формуле Тейлора с учетом того, что $\partial g(w, \zeta) / \partial s_{w}=0$ при $w \in \gamma$, получаем для $w^{*}=w_{t}, w \in \gamma$

$$
g\left(w^{*}, \zeta\right)=g(w, \zeta)+\frac{\partial g(w, \zeta)}{\partial n_{w}} \delta n_{w}+O\left(\varepsilon^{2}\right)=\frac{\partial g(w, \zeta)}{\partial n_{w}} \operatorname{Re}\left(e^{-i \alpha_{w}} t h(w)\right)+O\left(\varepsilon^{2}\right) .
$$

Для комплексной функции Грина $P(w, \zeta)$ справедливы соотношения

$$
P(w, \zeta)=-\ln (w-\zeta)+U(w, \zeta), \quad P^{\prime}(w, \zeta)=-\frac{1}{w-\zeta}+U^{\prime}(w, \zeta) .
$$

При $w \in \gamma, \zeta \in K$ имеем $|d w|=d s_{w}, d w=e^{i \beta_{w}} d s_{w}$, тем самым $d s_{w}=e^{-i \beta_{w}} d w \in \mathbb{R}$; кроме того,

$$
\frac{\partial g(w, z)}{\partial n_{w}}=P^{\prime}(w, z) e^{i \alpha_{w}}=i P^{\prime}(w, z) w^{\prime}>0
$$

и $\delta n_{w}=\operatorname{Re}\left(e^{-i \alpha_{w}} \Delta w\right)=\operatorname{Re}\left(e^{-i \alpha_{w}} t h(w)\right)$. Теперь снова для $w \in \gamma^{*}$ по граничным значениям $u_{0}(w)=\partial g(w, z) / \partial n_{w}=P^{\prime}(w, z) e^{i \alpha_{w}}$ на кривой $\gamma^{*}$ (см. соотношение $(8)$ ) с помощью формулы Грина построим гармоническую в области $G^{*}$ функцию $u(z)$ и учтем, что (см. (3) и (8))

$$
\begin{aligned}
\frac{\partial g(w, \zeta)}{\partial n_{w}} \frac{\partial g(w, z)}{\partial n_{w}} \delta n_{w} d s_{w} & =P^{\prime}(w, \zeta) e^{i \alpha_{w}} P^{\prime}(w, z) e^{i \alpha_{w}} \operatorname{Re}\left(e^{-i \alpha_{w}} \Delta w\right) e^{-i \beta_{w}} d w= \\
& =\operatorname{Re}\left\{P^{\prime}(w, \zeta) e^{i \alpha_{w}} P^{\prime}(w, z) e^{i \alpha_{w}} e^{-i \alpha_{w}} t h(w) e^{-i \beta_{w}} d w\right\}= \\
& =\operatorname{Re}\left\{i P^{\prime}(w, \zeta) P^{\prime}(w, z) \operatorname{th}(w) d w\right\}
\end{aligned}
$$

Рассуждая теперь аналогично разделу 1 и используя (9), окончательно получаем

$$
g^{*}(z, \zeta)-g(z, \zeta)=\operatorname{Re}\left\{\frac{t}{2 \pi i} \int_{\gamma} P^{\prime}(w, \zeta) P^{\prime}(w, z) h(w) d w\right\}+O\left(t^{2}\right), \quad|t|=\varepsilon
$$

(ср. с формулой (1)). Отметим, что в силу конформной инвариантности функции Грина формула (10) справедлива и для области $G$, содержащей бесконечно удаленную точку. 
Из (10) очевидным образом вытекает вариационная формула Адамара (1).

Получим теперь из равенства (10) вариационную формулу Шиффера (5). Пусть $z_{0} \in G, t=e^{i 2 \phi} \rho^{2}, h(z)=1 /\left(z-z_{0}\right), z^{*}=z_{t}=z+t h(z)$. Функция $h$ голоморфна в некоторой окрестности кривой $\gamma$, следовательно, мы можем воспользоваться равенством (10). Поскольку $P^{\prime}(w, z)=-1 /(w-z)+A(w, z)$, где функция $A=U^{\prime}$ голоморфна в $G$, подынтегральное выражение в (10) имеет в $G$ простые полюсы в точках $w=z, \zeta, z_{0}$ с вычетами $-1,-1,1$ соответственно. Следовательно, по теореме о вычетах

$$
\frac{1}{2 \pi i} \int_{\gamma} P^{\prime}(w, \zeta) P^{\prime}(w, z) h(w) d w=-\frac{P^{\prime}(\zeta, z)}{\zeta-z_{0}}-\frac{P^{\prime}(z, \zeta)}{z-z_{0}}+P^{\prime}\left(z_{0}, \zeta\right) P^{\prime}\left(z_{0}, z\right) .
$$

Непосредственно из (10) и (11) вытекает вариационная формула Шиффера (5).

Избавимся теперь от требования аналитичности границы $\gamma$. А именно, докажем формулу (5) для области $G$, граница которой состоит из конечного числа произвольных континуумов ${ }^{2)}$. Пусть $z, \zeta \in K \Subset G$ и пусть $\left\{G_{n}\right\}-$ последовательность областей с аналитическими границами, исчерпывающая область $G: \bar{G}_{n} \subset G_{n+1}$, $\bigcup_{n=1}^{\infty} G_{n}=G$. Для каждой из областей $G_{n}$ формула (5) справедлива равномерно по $z, \zeta \in K \Subset G$ и $z_{0} \in G$. Анализ доказательства, проведенного выше, показывает, что величины, входящие в остаточный член $O\left(t^{2}\right)$ в $(10)$, зависят только от интегралов от гармонических функций и их производных, связанных с областью $G_{n}$. В силу того что значения вышеуказанных интегралов от гармонических функций не зависят от кривых интегрирования, мы можем в процессе предельного перехода $G_{n} \rightarrow G$ при $n \rightarrow \infty$ зафиксировать кривые, по которым ведется интегрирование, и считать, например, что интегрирование ведется по границе $\gamma_{0}=\partial G_{0}$ некоторой начальной области $G_{0}$. Тогда при $G_{n} \rightarrow G$ (в вышеуказанном смысле) все гармонические функции для $G_{n}$, входящие в $O\left(t^{2}\right)$, вместе со всеми своими производными равномерно на $\gamma_{0}$ сходятся к соответствующим функциям для $G$, так что величина $O\left(t^{2}\right)$ остается равномерно ограниченной для $z, \zeta \in K$. Для этих же $z, \zeta, z_{0}$ величины, связанные с функцией Грина для области $G_{n}$, сходятся к соответствующим величинам для области $G$. Таким образом, из формулы $(5)$ для области $G_{n}$ путем предельного перехода мы получаем соответствующую вариационную формулу для функции Грина области $G$ (с заменой $O\left(\rho^{4}\right)$ на $\left.o\left(\rho^{2}\right)\right)$.

Пусть теперь вне $G$ заданы и зафиксированы некоторые точки $a_{1}, \ldots, a_{m}$ (например, все $\left.a_{k} \in \gamma\right)$. Положим

$$
A_{m}(z)=\prod_{k=1}^{m}\left(z-a_{k}\right), \quad q_{\mu}(z)=\prod_{j=0}^{\mu}\left(z-z_{j}\right)
$$

где $z_{0}, z_{1}, \ldots, z_{\mu}$ - произвольные различные точки области $G, \mu \geqslant m+1$. Пусть $h(z)=A_{m}(z) / q_{\mu}(z)$, тогда вариация $z \mapsto z_{t}=z+t h(z)$ удовлетворяет вышеперечисленным условиям и оставляет неподвижными точки $a_{1}, \ldots, a_{m}$. Такая вариация рассматривается как композиция вариаций Шиффера и естественным образом возникает при изучении проблемы модулей [16]. Из формулы (10), используя теорему

2) Ясно, что приводимые рассуждения, основанные на предельном переходе, справедливы для произвольной области $G$. 
о вычетах, получаем (ср. с равенством (6))

$$
g^{*}\left(z^{*}, \zeta^{*}\right)-g(z, \zeta)=\operatorname{Re}\left\{t \sum_{j=0}^{\mu} \frac{P^{\prime}\left(z_{j}, \zeta\right) P^{\prime}\left(z_{j}, z\right) A_{m}\left(z_{j}\right)}{q_{\mu}^{\prime}\left(z_{j}\right)}\right\}+O\left(t^{2}\right)
$$

при $z, \zeta \in K \Subset G$. С помощью предельного перехода, как и выше, устанавливается справедливость формулы (12) для конечносвязной области с произвольной границей (с заменой $O\left(t^{2}\right)$ на $o(t)$; разумеется так же, как и в работе [16], некоторые из точек $z_{j}$ можно было бы взять и вне области $G$, тогда в окончательной формуле $(12)$ они бы просто отсутствовали).

Пусть, наконец, все точки $z_{j}$ совпадают: $z_{j}=z_{0} \in G, j \geqslant 1$, и, соответственно, $q_{\mu}(z)=\left(z-z_{0}\right)^{\mu+1}$. Тогда вместо (12) получаем

$$
g^{*}\left(z^{*}, \zeta^{*}\right)-g(z, \zeta)=\frac{1}{\mu !} \operatorname{Re}\left\{\left.t\left(P^{\prime}(w, \zeta) P^{\prime}(w, z) A_{m}(w)\right)^{\mu}\right|_{w=z_{0}}\right\}+O\left(t^{2}\right)
$$

при $z, \zeta \in K \Subset G$. Справедливость данной формулы для конечносвязной области с произвольной границей устанавливается путем предельного перехода, как и выше. Вариация функции Грина вида (13) успешно применялась в различных задачах математической физики и теории аппроксимаций [12], [13], [20]-[22].

Отметим, что в стандартных обозначениях функционального анализа формула (10) принимает следующий вид (ср. с работой [6]):

$$
\delta_{\phi} g(z, \zeta)=\operatorname{Re}\left\{\frac{e^{i \phi}}{2 \pi i} \int_{\gamma} P^{\prime}(w, \zeta) P^{\prime}(w, z) h(w) d w\right\}
$$

где $t=\varepsilon e^{i \phi}, \delta_{\phi} g(z, \zeta)$ - вариация функции Грина “по направлению” $\phi$.

\section{4. ЗАКЛЮЧЕНИЕ}

Получена достаточно общая вариационная формула (10) для функции Грина, из которой вытекают, в частности, классические вариационные формулы Адамара (1) и Шиффера (5).

Благодарности. Автор поддержан РФФИ (грант № 11-01-00330_а) и Программой поддержки ведущих научных школ (грант НШ-8033.2010.1).

\section{Список литературы}

[1] J. Hadamard, "Mémoire sur le problème d'analyse relatif à l'équilibre des plaques élastiques encastrées", Mémoires des savants étrangers, 33, l'Acad. Sci., Paris, 1908, 1-128; Euvres de Jacques Hadamard, v. 2, CNRS, Paris, 1968, 515-631.

[2] M. Schiffer, Amer. J. Math., 68:3 (1946), 417-448.

[3] J. Hadamard, Euvres de Jacques Hadamard, tome II, Comité de publication des oeuvres de Jacques Hadamard, eds. M. Fréchet, P. Levy, S. Mandelbrojt, L. Schwartz, CNRS, Paris, 1968.

[4] S. E. Warschawski, J. Math. Mech., 9 (1960), 497-511.

[5] P. R. Garabedian, M. Schiffer, Trans. Amer. Math. Soc., 65 (1949), 187-238.

[6] М. Шиффер, "Некоторые новые результаты в теории конформных отображений", в кн.: Р. Курант, Принцип Дирихле, конформные отображения и минимальные поверхности, ИЛ, М., 1953, 234-301. 
[7] E. Schippers, W. Staubach, Israel J. Math., 173:1 (2009), 279-303.

[8] A. Marshakov, P. Wiegmann, A. Zabrodin, Commun. Math. Phys., 227:1 (2002), 131-153, arXiv: hep-th/0109048.

[9] I. Krichever, M. Mineev-Weinstein, P. Wiegmann, A. Zabrodin, Physica D, 198:1-2 (2004), $1-28$.

[10] I. Krichever, A. Marshakov, A. Zabrodin, Commun. Math. Phys., 259:1 (2005), 1-44, arXiv: hep-th/0309010.

[11] A. Zabrodin, Random matrices and Laplacian growth, arXiv:0907.4929, 2009.

[12] A. Martínez-Finkelshtein, E. A. Rakhmanov, "On asymptotic behavior of Heine-Stieltjes and Van Vleck polynomials", Recent Trends in Orthogonal Polynomials and Approximation Theory, Contemp. Math., 507, AMS, Providence, RI, 2010, 209-232.

[13] A. Martínez-Finkelshtein, E. Rakhmanov, Commun. Math. Phys., 302:1 (2011), 53-111, arXiv: 0902.0193.

[14] М. Шиффер, Д. К. Спенсер, Функиионалы на конечных римановых поверхностях, ИЛ, M., 1957.

[15] J. A. Jenkins, Ann. Math. (2), 66:3 (1957), 440-453.

[16] Г. В. Кузьмина, Тр. МИАН СССР, 139, 1980, 3-241.

[17] В. Н. Дубинин, УМН, 49:1(295) (1994), 3-76.

[18] В.Н. Дубинин, Емкости конденсаторов и симметризация в геометрической теории функиий комплексного переменного, Дальнаука, Владивосток, 2009.

[19] P. L. Duren, Univalent Functions, Fundamental Principles of Mathematical Sciences, 259, Springer, New York, 1983.

[20] S. Kamvissis, E. A. Rakhmanov, J. Math. Phys., 46:8 (2005), 083505, 24 pp.

[21] А. А. Гончар, Е. А. Рахманов, С. П. Суетин, УМН, 66:6(402) (2011), 3-36.

[22] А. Мартинес-Финкельштейн, Е.А. Рахманов, С.П. Суетин, УМН, 66:1(397) (2011), 183-184.

[23] А. Мартинес-Финкельштейн, Е. А. Рахманов, С. П. Суетин, Матем. сб., 202:12 (2011), 113-136.

[24] Е. А. Перевозникова, Е. А. Рахманов, Вариация равновесной энергии и $S$-свойство компактов минимальной емкости, Препринт, МИАН, М., 1994. 